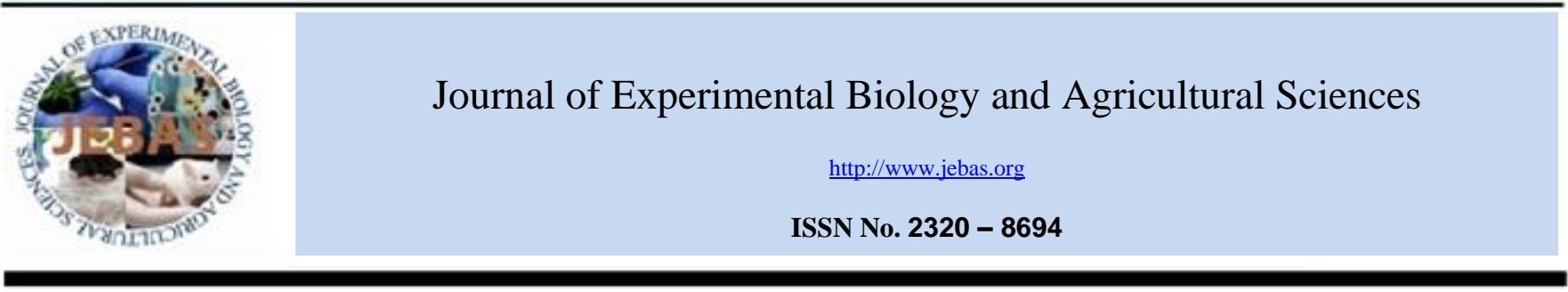

\title{
ADJUVANT IMPROVES THE EFFICACY OF HERBICIDE FOR WEED MANAGEMENT IN MAIZE SOWN UNDER ALTERED SOWING METHODS
}

\author{
Muhammad Javaid Akhter ${ }^{1}$, Rana Nadeem Abbas ${ }^{1}$, Muhammad Ahmed Waqas ${ }^{1}$, Mehmood Ali \\ Noor $^{2 *}$, Muhammad Awais Arshad ${ }^{1}$, Wajid Mahboob ${ }^{3}$, Faisal Nadeem ${ }^{1}$, Muhammad Azam ${ }^{1}$ and \\ Umair Gull ${ }^{1}$

\footnotetext{
${ }^{1}$ Department of Agronomy, University of Agriculture, Faisalabad-38040, Pakistan

${ }^{2}$ Institute of Crop Science, Chinese Academy of Agricultural Sciences, Key Laboratory of Crop Physiology and Ecology, Ministry of Agriculture, Beijing 100081, China
} \\ ${ }^{3}$ Plant Physiology Division, Nuclear Institute of Agriculture (NIA), Tandojam, 70050 Pakistan.
}

Received - December 30, 2016; Revision - January 14, 2017; Accepted - February 16, 2017

Available Online - February 28, 2017

DOI: http://dx.doi.org/10.18006/2017.5(1).022.030

KEYWORDS
Maize
Adjuvant
Sowing Method
Herbicide
Urea

\begin{abstract}
Field experiment was performed to investigate the effect of adjuvant on efficacy of herbicides for weed management in maize under different sowing methods. Urea as an adjuvant was used to increase the efficacy of atrazine plus mesotrion herbicide. Weed density, fresh and dry weight of weeds, nutrient uptake (NPK) by weeds, yield and yield related components of maize were significantly affected by sowing methods and different chemical control treatments. Biomass of weeds were decreased in most cases, weeds were controlled in almost all treatments, herbicides' full and reduced doses were not superior to manual hoeing treatments. Interactive effect among sowing methods and various chemical control treatments significantly affected the weed density, cob length, weight of 1000 grain, number of grains per cob, grain yield and biological yield. Among weed parameters, maximum weed density and biomass was observed at 20 and 40 days after sowing (DAS), and at harvesting when maize was sown as a flat sowing method and sprayed with Mesotrion + Atrazine at the rate of $19.76 \mathrm{~g}$ a.i. $\mathrm{ha}^{-1}+197.6 \mathrm{~g}$ a.i. $\mathrm{ha}^{-1}+3 \%$ Urea, while maximum economic yield $\left(7.51 \mathrm{t} \mathrm{ha}^{-1}\right)$ and biological yield $\left(21.82 \mathrm{t} \mathrm{ha}^{-1}\right)$ were found in ridge sown plots as compared to control treatment. The study suggested that the herbicide dose can be condensed up to $20 \%$ if urea solution is used as adjuvant to get the same effectiveness as with recommended dose, with no compromised maize yield.
\end{abstract}

* Corresponding author

E-mail: mehmood2017@gmail.com (Mehmood Ali Noor)

Peer review under responsibility of Journal of Experimental Biology and Agricultural Sciences.

Production and Hosting by Horizon Publisher India [HPI] (http://www.horizonpublisherindia.in/).

All rights reserved.
All the article published by Journal of Experimental Biology and Agricultural Sciences is licensed under a Creative Commons Attribution-NonCommercial 4.0 International License Based on a work at www.jebas.org.

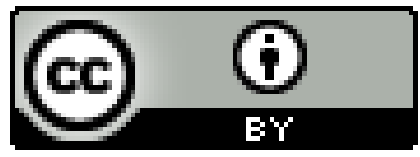




\section{Introduction}

Worldwide a very large sum of maize productivity, nearly $40 \%$ reduced as result of weeds infestation (Oerke \& Dehne, 2004). Major weeds of maize are Trianthema partulacastrum, Cyperus rotundus and Cynodon dactylon. These weeds compete with maize plants for nutrient, moisture, light and space, this influx of weed leads to compromise net profit and sometime total failure of crop. Weeds also act as the host for many insects and pests causing major diseases and troublesome during cultural practices, ultimately production cost exceeds. Weeds can be controlled by non-chemical (cultural, mechanical, biological) and chemical methods. No doubt non-chemical weed control methods are still useful, but are time consuming, laborious and expensive (Chikoye et al., 2004). In maize crop chemical weed control is the most effective and economical alternative to reduce weed infestation in order to get healthy crop stand.

Herbicides have become a vital component for weed management. These not only control the weeds and increase yield but also saves energy, labour, time and reduce erosion and farming cost (Anonymous, 2006). Asia and United States alone consume $40 \%$ and $32 \%$ of world herbicides, respectively (FAO, 2011). There are many drawbacks of herbicide applications especially environmental hazards due to poor application performance (Pacanoski, 2007). Climatic extremes, environmental pollution and their associated risks regarding human health are major concerns in modern agriculture (Afzal et al., 2015). Therefore, it is a dire need of time to develop novel techniques that can reduce chemical load in agro ecosystems through decreasing dose and spray volume of the chemicals. This may be possible by optimizing efficiency of herbicides at reduced rates by addition of adjuvant (Devendra et al., 2004).

An adjuvant is a biologically active substance, added to herbicide formulation to enhance the effectiveness of herbicide by decreasing the surface tension, beading and bouncing off of droplets and provides a better coverage of plant (Green \& Beestman, 2007). Use of adjuvant along the herbicide increases the efficacy and retention of herbicide through cuticle. The action of adjuvant comprises retention of spray droplet, surface wet ability and absorption of herbicide (Bunting et al., 2004). Adjuvant includes surfactants, oil and fertilizers. Selection of adjuvant depends on herbicide used, presence of weeds and environment. Urea is a fertilizer, which can effectively be used with herbicide as an adjuvant for controlling weeds as it enhanced the efficacy and penetration of herbicide to reduce the weed biomass (Ssango \& Balitenda, 2003; Bunting et al., 2004). Nitrogen fertilizers as an adjuvant increase the phytotoxic effect of foramsulfuron in maize crop (Bunting et al., 2004). Similarly, Getmanetz et al. (1991) reported that herbicide application along with urea as an adjuvant provided $12-13.5 \%$ better control as compared to when herbicide applied alone.
Likewise, adaptation of site specific and optimal sowing method is also a perquisite to ensure control over weed infestation (Ullah et al., 2017). Appropriate sowing method has many advantages due to easy inter-row operations, uniform irrigation, weed and pest management and mechanical harvesting (Memon et al., 2007). Adaptation of inappropriate sowing method and time can lead to unproductive plants, low crop yield and even to barren lands (Ahmad et al., 2016; Bakht et al., 2011). Different sowing methods are used for maize in the world to provide optimum microclimate to control weeds. Sowing methods which are mostly being used in Pakistan are conventional sowing, flat sowing and bed sowing methods (Amin et al., 2006). Optimal sowing method at proper sowing time is a key factor to get the maximum potential and profitability of maize hybrids (Alias et al., 2003; Bakhtavar et al., 2015). This multidimensional study was conducted to evaluate the effectiveness of reduced rates of herbicide by adjuvant mixing to manage weeds under different sowing methods in maize crop. Variations in macro nutrients uptake by weeds under altered sowing methods were also analyzed.

\section{Materials and Methods}

\subsection{Experimental detail}

Planned experiment was conducted to evaluate the effects of adjuvant on efficacy of herbicides for weed management in maize under different sowing methods. Experiment was performed at Student Farm Department of Agronomy, University of Agriculture, Faisalabad, Pakistan during summer season of 2014. Before crop sowing the field has large infestation of weeds. Climatic parameters studied during experiment were given in figure 1. Study was conducted in randomized complete block design (RCBD) using split plot arrangement with three replications. Each plot having size of 7 $\mathrm{m} \times 2.4 \mathrm{~m}$. It was two factor study i.e. sowing methods (Ridge Sowing, Flat Sowing, Bed Sowing) were kept in main plots and different levels of herbicides with adjuvant, $\mathrm{T}_{0}=$ Control (two hoeing's), $\mathrm{T}_{1}=$ Mesotrion + Atrazine @ $49.4 \mathrm{~g}$ a.i. ha ${ }^{-1}+$ $494 \mathrm{~g}$ a.i. ha ${ }^{-1}$ (Recommended), $\mathrm{T}_{2}=$ Mesotrion + Atrazine @ 39.52 g a.i. ha ${ }^{-1}+395.2$ g a.i. ha ${ }^{-1}+3 \%$ Urea, $\mathrm{T}_{3}=$ Mesotrion + Atrazine @ 29.64 g a.i. ha ${ }^{-1}+296.4$ g a.i. ha ${ }^{-1}+3 \%$ Urea, $\mathrm{T}_{4}=$ Mesotrion + Atrazine @ 19.76 g a.i. ha ${ }^{-1}+197.6$ g a.i. ha $^{-1}+$ $3 \%$ Urea, were kept in sub plots.

\subsection{Herbicide application protocol}

Pre mixed herbicide atrazine + mesotrione along with adjuvant was applied at 2-3 leaf stage of weeds with dose as decided. Herbicide was applied by "Knapsack" sprayer fitted with flatfan nozzle. Total volume of water used for spraying was $300 \mathrm{~L}$ $\mathrm{ha}^{-1}$, spray volume was determined by standard calibration before herbicide spray. 


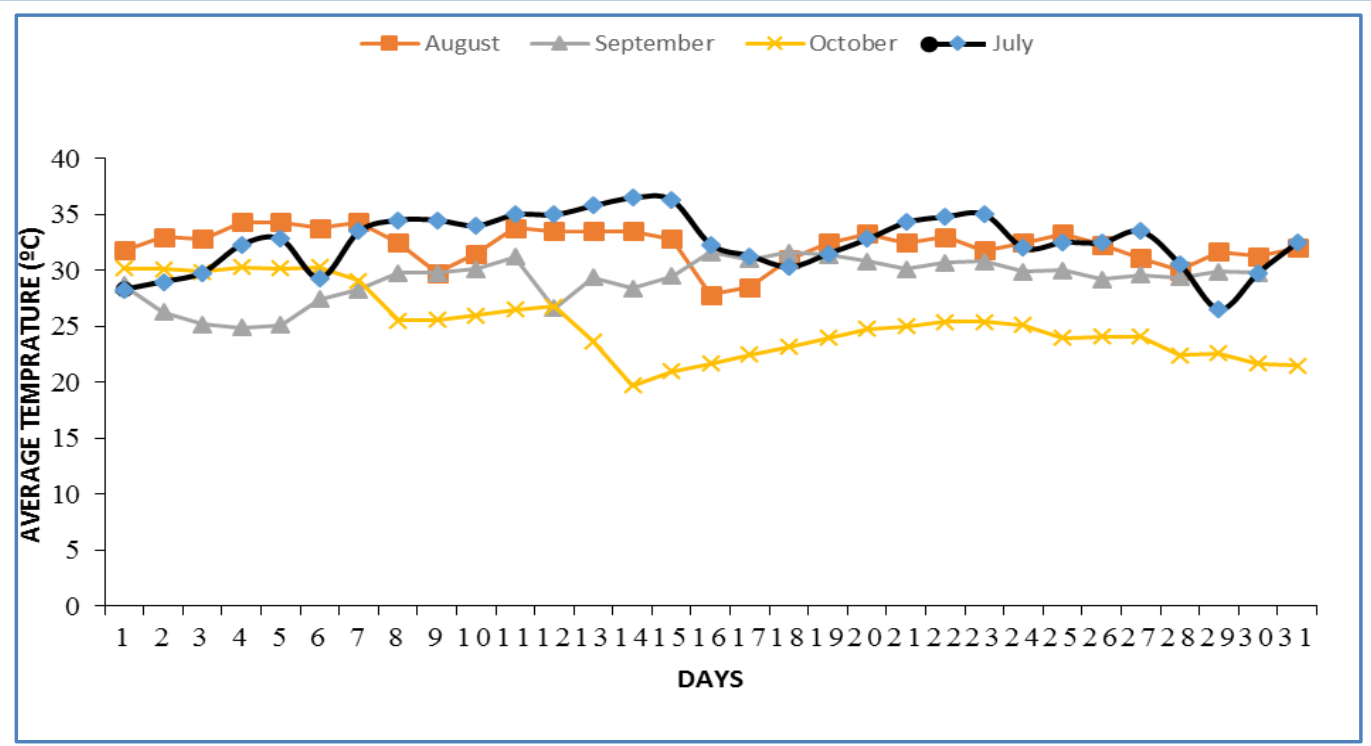

Figure 1 Meteorological data on weekly average temperature $\left({ }^{\circ} \mathrm{C}\right)$ during the course of present study (July-September) collected from Meteorological Observatory, Department of Crop Physiology, University of Agriculture, Faisalabad.

\subsection{Plant material and crop husbandry}

Maize hybrid Monsanto DK-919 was purchased from Monsanto Pakistan (Pvt) Ltd. Seeds were sown in $4^{\text {th }}$ week of July, with seed rate of $25 \mathrm{~kg} \mathrm{ha}^{-1}$ having plant to plant distance of $20 \mathrm{~cm}$ and row to row distance of $60 \mathrm{~cm}$, full dose of phosphorus and potassium and $1 / 3$ of nitrogen was applied at seedbed preparation and remaining nitrogen was applied in split doses. Two manual hoeings at 15 and 30 DAS were done in control treatment; all other treatments availed same and standard agronomic practices.

\subsection{Measurements}

Data concerning weed density, weeds biomass and plant population was recorded from an area of $1 \mathrm{~m}^{2}$ from three randomly selected areas. From each plot ten plants were selected randomly to record plant height, cob length, 1000grain weight, number of grains per cob, number of cobs per plant, number of rows per cob, while biological yield, grain yield and harvest index were recorded by harvesting whole plot. Harvest index was determined from grain yield and biological yield using formula as:

$$
\text { Harvest index }(\%)=\frac{\text { Economic yield }}{\text { Biological yield }} \times 100
$$

\subsection{Biochemical analysis of weeds}

For digestion, weed samples were oven dried at $70^{\circ} \mathrm{C}$ till constant weight and finely ground by using an electric grinder. Weighed $0.1 \mathrm{~g}$ of dry ground sample and each added into suitable conical flasks $(50 \mathrm{ml})$. It was followed by the mixing of $2.5 \mathrm{ml}$ of concentrated $\mathrm{H}_{2} \mathrm{SO}_{4}$ into each flask containing weighed plant sample and left overnight at $26^{\circ} \mathrm{C}$. Later on $1 \mathrm{ml}$ of $\mathrm{H}_{2} \mathrm{O}_{2}$ added into each flask (containing plant sample + $\mathrm{H}_{2} \mathrm{SO}_{4}$ which were left for overnight) and after that flasks were transferred to hot plate placed in a fume hood for heating. Samples were heated at $200^{\circ} \mathrm{C}$ for 30 minutes during which fumes of $\mathrm{H}_{2} \mathrm{O}_{2}$ began to come out from flasks. After that, temperature of hot plate was increased to $250^{\circ} \mathrm{C}$ for next 30 minutes, during which digested material became completely colorless. Digested material was filtered and distilled water was added up to the final volume of $30 \mathrm{ml}$. Aliquots were then stored for the determination of nitrogen $(\mathrm{N})$, phosphorus $(\mathrm{P})$ and potassium $(\mathrm{K})$ by various methods. $\mathrm{N}$ was determined by micro-Kjeldhal method (Bremner, 1965).

Nitrogen was calculated by equation:

$$
\text { Nitrogen } \%=(T \times N \times 1.4) / \text { Sample weight }
$$

Where,

$\mathrm{T}=$ Volume of acid used for titration $(\mathrm{ml})$

$\mathrm{N}=$ Normality of acid $=0.01 \mathrm{~N}$

Sample weight $=0.1 \mathrm{~g}$

Whereas, phosphorus in weed samples was measured by method given by Wolf (1982). For that, the digested material $(5 \mathrm{ml})$ was dissolved in $10 \mathrm{ml}$ Barton reagent and volume was made up to $50 \mathrm{ml}$. The samples were kept for half an hour and phosphorus contents were determined with an ANA-730 Spectrophotometer at wavelength of $470 \mathrm{~nm}$ after calibration with $\mathrm{P}$ standards. Potassium was determined with flame photometer (Jenway PFP7). A graded series of standards (ranging from 2-20 ppm) of $\mathrm{K}$ using $\mathrm{AR} \mathrm{KCl}$ were prepared and standard curve was drawn. The values of plant $\mathrm{K}$ were determined from standard curve. 
Table 1 Effect of herbicide with adjuvant on weed density $\left(\mathrm{m}^{-2}\right)$ at harvest in maize under different sowing methods.

\begin{tabular}{lc} 
Treatments & \multicolumn{1}{c}{ T. } \\
& 20 DAS \\
$\mathrm{T}_{0} \times \mathrm{M}_{1}$ & $54.00 \pm 7.57^{\mathrm{h}}$ \\
$\mathrm{T}_{1} \times \mathrm{M}_{1}$ & $82.67 \pm 5.48^{\mathrm{fg}}$ \\
$\mathrm{T}_{2} \times \mathrm{M}_{1}$ & $81.33 \pm 2.84^{\mathrm{fg}}$ \\
$\mathrm{T}_{3} \times \mathrm{M}_{1}$ & $112.00 \pm 5.50^{\mathrm{e}}$ \\
$\mathrm{T}_{4} \times \mathrm{M}_{1}$ & $142.67 \pm 6.88^{\mathrm{d}}$ \\
$\mathrm{T}_{0} \times \mathrm{M}_{2}$ & $88.67 \pm 3.75^{\mathrm{efg}}$ \\
$\mathrm{T}_{1} \times \mathrm{M}_{2}$ & $183.33 \pm 4.096^{\mathrm{c}}$ \\
$\mathrm{T}_{2} \times \mathrm{M}_{2}$ & $182.00 \pm 19.03^{\mathrm{c}}$ \\
$\mathrm{T}_{3} \times \mathrm{M}_{2}$ & $205.33 \pm 8.08^{\mathrm{b}}$ \\
$\mathrm{T}_{4} \times \mathrm{M}_{2}$ & $234.00 \pm 4.04^{\mathrm{a}}$ \\
$\mathrm{T}_{0} \times \mathrm{M}_{3}$ & $71.00 \pm 2.64^{\mathrm{gh}}$ \\
$\mathrm{T}_{1} \times \mathrm{M}_{3}$ & $99.67 \pm 4.09^{\mathrm{ef}}$ \\
$\mathrm{T}_{2} \times \mathrm{M}_{3}$ & $98.67 \pm 7.51^{\mathrm{ef}}$ \\
$\mathrm{T}_{3} \times \mathrm{M}_{3}$ & $107.67 \pm 14.51^{\mathrm{ef}}$ \\
$\mathrm{T}_{4} \times \mathrm{M}_{3}$ & $146.00 \pm 12.74^{\mathrm{d}}$ \\
$\mathrm{LSD}$ & 30.281
\end{tabular}

\section{T. portulacastrum}

40 DAS

$35.67 \pm 4.84^{\mathrm{i}}$

$40.67 \pm 2.18^{\mathrm{hi}}$

$38.33 \pm 3.382^{\mathrm{i}}$

$74.33 \pm 12.44^{\mathrm{de}}$

$95.67 \pm 9.024^{\mathrm{bc}}$

$60.67 \pm 5.23^{\mathrm{efgh}}$

$69.33 \pm 8.87^{\mathrm{def}}$

$62.00 \pm 2.64^{\mathrm{efg}}$

$112.00 \pm 8.02^{\mathrm{b}}$

$234.00 \pm 10.14^{\mathrm{a}}$

$45.00 \pm 3.78^{\text {ghi }}$

$49.67 \pm 13.54^{\text {fghi }}$

$46.00 \pm 2.51^{\text {ghi }}$

$72.33 \pm 6.69^{\mathrm{de}}$

$89.33 \pm 9.49^{\mathrm{cd}}$

21.108

\section{C. rotundus}

20 DAS

40 DAS

$13.00 \pm 4.35^{\mathrm{h}}$

$22.67 \pm 1.45 \mathrm{fg}$

$20.00 \pm 0.57^{\text {gh }}$

$33.67 \pm 2.90^{\text {cde }}$

$46.00 \pm 2.51^{\mathrm{ab}}$

$23.33 \pm 1.20^{\mathrm{fg}}$

$33.33 \pm 2.60^{\text {cde }}$

$29.33 \pm 0.88^{\text {ef }}$

$40.33 \pm 1.33^{\mathrm{bc}}$

$52.67 \pm 2.72^{a}$

$19.67 \pm 3.38^{\text {gh }}$

$30.00 \pm 1.52^{\text {def }}$

$29.00 \pm 2.30^{\mathrm{ef}}$

$37.00 \pm 4.17^{\mathrm{cd}}$

$49.33 \pm 0.89^{\mathrm{a}}$

7.5870
$12.66 \pm 0.33^{\mathrm{i}}$

$15.66 \pm 1.20^{\mathrm{hi}}$

$14.33 \pm 1.20^{\mathrm{i}}$

$28.00 \pm 3.21^{\text {de }}$

$41.00 \pm 2.51^{\mathrm{b}}$

$21.33 \pm 0.88^{\mathrm{fg}}$

$25.33 \pm 0.89^{\mathrm{ef}}$

$23.33 \pm 1.20^{\text {efg }}$

$35.66 \pm 1.66^{\mathrm{c}}$

$47.66 \pm 2.72^{\mathrm{a}}$

$20.00 \pm 1.73^{\text {gh }}$

$23.33 \pm 0.33^{\text {efg }}$

$22.66 \pm 1.20^{\mathrm{fg}}$

$32.00 \pm 4.16^{\mathrm{cd}}$

$44.33 \pm 0.88^{\mathrm{ab}}$

5.2496

Means which followed by the same letters within a column are not significantly different at $P \leq 0.05$. $M_{1}=$ Ridge Sowing, M $M_{2}=$ Flat Sowing, $M_{3}=$ Bed Sowing; $T_{0}=$ Control (two manual hoeings); $T_{1}=$ Mesotrion + atrazine @ $49.4 \mathrm{~g}$ a.i. ha $a^{-1}+494 \mathrm{~g}$ a.i. ha ${ }^{-1}$ (Recommended dose); $T_{2}=$ Mesotrion + atrazine @ $39.52 \mathrm{~g}$ a.i.ha $a^{-1}+395.2 \mathrm{~g}$ a.i.ha $\mathrm{a}^{-1}+3 \%$ Urea; $T_{3}=$ Mesotrion + atrazine @ 29.64 g a.i. ha $a^{-1}+296.4$ g a.i. ha $a^{-1}+3 \%$ Urea; $T_{4}=$ Mesotrion + atrazine @ 19.76 g a.i. ha $a^{-1}+197.6$ g a.i. ha $a^{-1}+3 \%$ Urea.

\subsection{Soil analysis}

Before sowing of maize, soil samples were collected from experimental site from depth of $30 \mathrm{~cm}$ and were analyzed by adopting standard procedures at the Soil Science department, University of Agriculture, Faisalabad. Laboratory investigations has revealed that soil was loamy with $0.58 \%$ organic matter, EC $0.44 \mathrm{dS} \mathrm{m}^{-1}, \mathrm{pH} 8.2$, Available NPK was $0.0352 \%, 4.5 \mathrm{mg} \mathrm{kg}^{-1}, 110 \mathrm{mg} \mathrm{kg}^{-1}$ respectively, and Bulk density (BD) was $1.40 \mathrm{~cm}^{-3}$.

\subsection{Statistical analysis}

The data obtained were analyzed statistically by using fisher's analysis of variance technique (ANOVA) following the described method by Steel et al. (1997). Least Significance Difference (LSD) test at 5\% probability level was employed to compare the significant difference among treatment's mean.

\section{Results}

\subsection{Weeds density and weed biomass bioassay}

Horse purslane ( $T$. portulacastrum L.) and purple nutsedge ( $C$. rotundus $\mathrm{L}$.) are the weed species that were found dominantly in the experimental area. All weed control treatments and different sowing methods significantly affect the weed density $\left(\mathrm{m}^{-2}\right)$, and their interactive effects were also observed in this study (Table 1).

After 20 and 40 days of sowing highest density of $T$. portulacastrum was observed in plots where maize was planted with flat sown technique and sprayed with Mesotrion + Atrazine @ 19.76 g a.i. $\mathrm{ha}^{-1}+197.6 \mathrm{~g}$ a.i. $\mathrm{ha}^{-1}+3 \%$ Urea. It was followed by the treatments having flat sown plots in combination with Mesotrion + atrazine @ 29.64 g a.i. ha ${ }^{-1}+$ 296.4 g a.i. ha ${ }^{-1}+3 \%$ Urea. Among various sowing methods lowest weed density was documented where maize was planted with ridge sowing method and weed, were controlled by two manual hoeing's after fifteen and thirty days of sowing. These results were statistically equal to plots where maize was planted with ridge sown technique and sprayed with Mesotrion + Atrazine @ 39.52 g a.i. ha ${ }^{-1}+395.2$ g a.i. ha $^{-1}+3 \%$ Urea.

Highest presence of $C$. rotundus was also reported after 20 and 40 days of sowing where maize crop was seeded with flat sown technique and sprayed with Mesotrion + Atrazine @ $19.76 \mathrm{~g}$ a.i. $\mathrm{ha}^{-1}+197.6 \mathrm{~g}$ a.i. $\mathrm{ha}^{-1}+3 \%$ Urea concentration. These results are not statistically different to plots where maize was planted with bed sowing method and sprayed with Mesotrion + atrazine @ $19.76 \mathrm{~g}$ a.i. $\mathrm{ha}^{-1}+197.6 \mathrm{~g}$ a.i. $\mathrm{ha}^{-1}+3 \%$ Urea, after 20 and 40 days of sowing. Most effective combo (having least weeds) was the treatment in which maize was sown with ridge sowing method and weeds were controlled with two manual hoeing's during crop season at 15 and 30 days after sowing (Table 1). 
Table 2 Effect of herbicide with adjuvant on weed biomass ( $\mathrm{g}$ $\mathrm{m}^{-2}$ ) at harvest in maize under different sowing methods.

$\begin{array}{lll}\text { Treatments } & \begin{array}{l}\text { Total weed fresh } \\ \text { weight }\left(\mathrm{g} \mathrm{m}^{-2}\right)\end{array} & \begin{array}{l}\text { Total weed dry } \\ \text { weight }\left(\mathrm{g} \mathrm{m}^{-2}\right)\end{array} \\ \mathrm{M}_{1} & 141.72 \pm 2.61^{\mathrm{c}} & 27.92 \pm 1.40^{\mathrm{c}} \\ \mathrm{M}_{2} & 236.64 \pm 5.32^{\mathrm{a}} & 46.78 \pm 2.22^{\mathrm{a}} \\ \mathrm{M}_{3} & 179.57 \pm 7.23^{\mathrm{b}} & 34.62 \pm 2.14^{\mathrm{b}} \\ \mathbf{L S D} & \mathbf{2 0 . 9 4} & \mathbf{4 . 8 6} \\ \mathrm{T}_{0} & 133.69 \pm 3.43^{\mathrm{d}} & 26.29 \pm 2.98^{\mathrm{d}} \\ \mathrm{T}_{1} & 171.88 \pm 5.34^{\mathrm{c}} & 33.35 \pm 1.56^{\mathrm{c}} \\ \mathrm{T}_{2} & 168.27 \pm 4.98^{\mathrm{c}} & 32.63 \pm 1.94^{\mathrm{c}} \\ \mathrm{T}_{3} & 210.60 \pm 4.56^{\mathrm{b}} & 41.56 \pm 3.42^{\mathrm{b}} \\ \mathrm{T}_{4} & 245.45 \pm 6.20^{\mathrm{a}} & 48.47 \pm 3.87^{\mathrm{a}} \\ \text { LSD } & 16.822 & 3.0264 \\ \text { Interaction } & \mathbf{N S} & \mathbf{N S}\end{array}$

Means which followed by the same letters within a column are not significantly different at $P \leq 0.05 . M_{1}=$ Ridge Sowing, $M_{2}$ $=$ Flat Sowing, $M_{3}=$ Bed Sowing; $T_{0}=$ Control (two manual hoeings); $T_{l}=$ Mesotrion + atrazine @ $49.4 \mathrm{~g}$ a.i. $\mathrm{ha}^{-1}+494 \mathrm{~g}$ a.i. hal (Recommended dose); $T_{2}=$ Mesotrion + atrazine @ 39.52 g a.i. $h a^{-1}+395.2$ g a.i. $h a^{-1}+3 \%$ Urea $; T_{3}=$ Mesotrion + atrazine@29.64 g a.i.ha $a^{-1}+296.4$ g a.i. $h a^{-1}+3 \%$ Urea; $T_{4}=$ Mesotrion + atrazine @ $19.76 \mathrm{~g}$ a.i. $h a^{-1}+197.6 \mathrm{~g}$ a.i. $h a^{-1}+3 \%$ Urea.

Weed biomass $\left(\mathrm{m}^{-2}\right)$ was also significantly decreased by all weed control treatments and different sowing methods during the study. Furthermore, interactive effects of sowing methods and weed control practices were non-significant (Table 2). Data pertaining to the weed biomass at crop harvesting revealed that among sowing methods flat sown plots of maize showed highest fresh and dry weight of weed and it was followed by bed sowing while, ridge sowing proved to be most effective to reduce the weed fresh and dry weight. Furthermore, in chemical weed control treatments application of Mesotrion + Atrazine @ $19.76 \mathrm{~g}$ a.i. ha ${ }^{-1}+197.6 \mathrm{~g}$ a.i. ha ${ }^{-1}+$ $3 \%$ Urea at crop harvest showed maximal values of total fresh and dry weed biomass, it was followed by Mesotrion + atrazine @ $29.64 \mathrm{~g}$ a.i. ha ${ }^{-1}+296.4 \mathrm{~g}$ a.i. ha ${ }^{-1}+3 \%$ Urea. Moreover, highest dose of Mesotrion + atrazine @ $49.4 \mathrm{~g}$ a.i. $\mathrm{ha}^{-1}+494 \mathrm{~g}$ a.i. ha ${ }^{-1}$ and Mesotrion + atrazine @ $39.52 \mathrm{~g}$ a.i. $\mathrm{ha}^{-1}+395.2 \mathrm{~g}$ a.i. ha ${ }^{-1}+3 \%$ Urea were found statistically equal in reducing the fresh and dry weight of weeds. In various sowing methods, least fresh and dry weed biomass were recorded from the two manual hoeing's plot.

\subsection{Biochemical analysis of weeds for mineral nutrients uptake}

Competition exists between maize crop and weed plants (weed-maize) for nitrogen, phosphorus $\left(\mathrm{P}_{2} \mathrm{O}_{5}\right)$ and potassium. Weeds mostly exhibit more power to absorb mineral nutrients than crop plants. Various sowing techniques (ridge, bed and flat sowing) adapted to maize plant in field and combination of various chemical and non-chemical (manual hoeings) in weed control practices significantly decrease the strength of weeds to absorb mineral nutrients (Table 3). But interaction between sowing methods and weed control practices was found nonsignificant. In those plots, where maize was sown with flat sown technique weeds flora exhibits higher competition to maize plants and weeds absorb highest amounts of mineral nutrients (nitrogen, phosphorous and potassium).

Table 3 Influence of weed control treatments on N, P and K $(\mathrm{kg} / \mathrm{ha})$ uptake by weeds under altering sowing methods

$\begin{array}{llll}\text { Treatments } & \mathbf{N}(\mathbf{k g} / \mathbf{h a}) & \mathbf{P}(\mathbf{k g} / \mathbf{h a}) & \mathbf{K}(\mathbf{k g} / \mathbf{h a}) \\ \mathrm{M}_{1} & 2.90 \pm 0.06^{\mathrm{c}} & 3.46 \pm 0.08^{\mathrm{b}} & 3.81 \pm 0.11^{\mathrm{c}} \\ \mathrm{M}_{2} & 5.06 \pm 0.19^{\mathrm{a}} & 7.33 \pm 1.06^{\mathrm{a}} & 6.72 \pm 1.96^{\mathrm{a}} \\ \mathrm{M}_{3} & 3.60 \pm 0.12^{\mathrm{b}} & 4.81 \pm 0.26^{\mathrm{b}} & 4.99 \pm 0.86^{\mathrm{b}} \\ \mathbf{L S D} & \mathbf{0 . 7 0} & \mathbf{1 . 5 2} & \mathbf{0 . 7 5} \\ \mathrm{T}_{0} & 2.57 \pm 0.09^{\mathrm{d}} & 2.81 \pm 0.16^{\mathrm{d}} & 3.53 \pm 0.33^{\mathrm{d}} \\ \mathrm{T}_{1} & 3.63 \pm 0.22^{\mathrm{c}} & 3.98 \pm 0.71^{\mathrm{cd}} & 4.69 \pm 0.09^{\mathrm{c}} \\ \mathrm{T}_{2} & 3.52 \pm 0.11^{\mathrm{c}} & 4.46 \pm 0.14^{\mathrm{c}} & 4.69 \pm 0.22^{\mathrm{c}} \\ \mathrm{T}_{3} & 4.39 \pm 0.27^{\mathrm{b}} & 6.50 \pm 1.52^{\mathrm{b}} & 5.97 \pm 0.66^{\mathrm{b}} \\ \mathrm{T}_{4} & 5.16 \pm 0.53^{\mathrm{a}} & 8.25 \pm 0.16^{\mathrm{a}} & 6.99 \pm 0.22^{\mathrm{a}} \\ \text { LSD } & \mathbf{0 . 6 1 2 3} & \mathbf{1 . 1 8 7 7} & \mathbf{0 . 6 3 4 5} \\ \text { Interaction } & \mathbf{N S} & \mathbf{N S} & \mathbf{N S}\end{array}$

Means which followed by the same letters within a column are not significantly different at $P \leq 0.05 ; M_{1}=$ Ridge Sowing, $M_{2}$ $=$ Flat Sowing, $M_{3}=$ Bed Sowing; $T_{0}=$ Control (two manual hoeings); $T_{1}=$ Mesotrion + atrazine @ $49.4 \mathrm{~g}$ a.i. $h a^{-1}+494 \mathrm{~g}$ a.i. ha ${ }^{-1}$ (Recommended dose); $T_{2}=$ Mesotrion + atrazine @ 39.52 g a.i. $h a^{-1}+395.2$ g a.i. $h a^{-1}+3 \%$ Urea; $T_{3}=$ Mesotrion + atrazine @ 29.64 g a.i. ha $a^{-1}+296.4$ g a.i. $h a^{-1}+3 \%$ Urea; $T_{4}=$ Mesotrion + atrazine @ 19.76 g a.i. ha $a^{-1}+197.6$ g a.i. $h a^{-1}+3 \%$ Urea.

Least weed-maize plants competition was observed in ridge sown plots for mineral nutrients (nitrogen, phosphorous and potassium). Among various weed control practices, maximal absorption of nitrogen, phosphorous $\left(\mathrm{P}_{2} \mathrm{O}_{5}\right)$ and potassium by weed flora was observed in those plots where Mesotrion + Atrazine@ $19.76 \mathrm{~g}$ a.i. $\mathrm{ha}^{-1}+197.6 \mathrm{~g}$ a.i. $\mathrm{ha}^{-1}+3 \%$ Urea were sprayed. Lowest weed-maize plants competition concerning with absorption of mineral nutrients (nitrogen, phosphorous and potassium) by weed was found in plots where nonchemical (two manual hoeing's at 15 and 30 DAS) method was practiced to control weed interference. This result was found statistically similar to plots sprayed with Mesotrion + atrazine @ 49.4 g a.i. ha ${ }^{-1}+494$ g a.i. ha ${ }^{-1}$ for phosphorous uptake.

\subsection{Agronomic traits}

Various sowing techniques (ridge, flat, and bed swing) adapted to maize plant and all manual and chemical weed control treatments significantly influenced cob length, 1000-grain weight, grains number per cob, economic and biological yield. Similarly, interactive effects were also significant. 
Table 4 Effect of herbicide with adjuvant on agronomic attributes of maize under different sowing methods.

$\begin{array}{lc}\text { Treatments } & \begin{array}{c}\text { Cob length } \\ (\mathbf{c m})\end{array} \\ \mathrm{T}_{0} \times \mathrm{M}_{1} & 19.04 \pm 0.11^{\mathrm{a}} \\ \mathrm{T}_{1} \times \mathrm{M}_{1} & 18.66 \pm 0.07^{\mathrm{b}} \\ \mathrm{T}_{2} \times \mathrm{M}_{1} & 18.71 \pm 0.04^{\mathrm{ab}} \\ \mathrm{T}_{3} \times \mathrm{M}_{1} & 17.63 \pm 0.06^{\mathrm{cd}} \\ \mathrm{T}_{4} \times \mathrm{M}_{1} & 16.97 \pm 0.09^{\mathrm{e}} \\ \mathrm{T}_{0} \times \mathrm{M}_{2} & 17.76 \pm 0.08 \mathrm{c} \\ \mathrm{T}_{1} \times \mathrm{M}_{2} & 17.20 \pm 0.2^{\mathrm{de}} \\ \mathrm{T}_{2} \times \mathrm{M}_{2} & 17.19 \pm 0.29^{\mathrm{de}} \\ \mathrm{T}_{3} \times \mathrm{M}_{2} & 16.31 \pm 0.10^{\mathrm{fg}} \\ \mathrm{T}_{4} \times \mathrm{M}_{2} & 14.89 \pm 0.14^{\mathrm{h}} \\ \mathrm{T}_{0} \times \mathrm{M}_{3} & 18.21 \pm 0.29^{\mathrm{bc}} \\ \mathrm{T}_{1} \times \mathrm{M}_{3} & 16.93 \pm 0.17^{\mathrm{e}} \\ \mathrm{T}_{2} \times \mathrm{M}_{3} & 16.72 \pm 0.08^{\mathrm{ef}} \\ \mathrm{T}_{3} \times \mathrm{M}_{3} & 16.21 \pm 0.15^{\mathrm{g}} \\ \mathrm{T}_{4} \times \mathrm{M}_{3} & 14.91 \pm 0.12^{\mathrm{h}} \\ \mathbf{L S D} & \mathbf{0 . 5 8 0 3}\end{array}$

\begin{tabular}{c}
$\begin{array}{c}\text { No. of grains } \\
\text { per cob }\end{array}$ \\
$602.53 \pm 4.15^{\mathrm{a}}$ \\
$580.67 \pm 8.09^{\mathrm{b}}$ \\
$586.67 \pm 7.33^{\mathrm{b}}$ \\
$563.53 \pm 3.46^{\mathrm{c}}$ \\
$474.73 \pm 2.54^{\mathrm{h}}$ \\
$532.60 \pm 6.77^{\mathrm{de}}$ \\
$505.13 \pm 4.60^{\mathrm{g}}$ \\
$511.00 \pm 4.36^{\mathrm{fg}}$ \\
$448.87 \pm 4.64^{\mathrm{i}}$ \\
$377.13 \pm 8.66^{\mathrm{j}}$ \\
\hline $554.07 \pm 6.17^{\mathrm{cd}}$ \\
\hline $528.07 \pm 7.16^{\mathrm{efg}}$ \\
\hline $531.27 \pm 6.64^{\mathrm{ef}}$ \\
$477.47 \pm 2.88^{\mathrm{h}}$ \\
$429.00 \pm 5.11^{\mathrm{i}}$ \\
223.502
\end{tabular}

$\begin{gathered}\text { 1000-grain weight } \\ (\mathbf{g})\end{gathered}$
$255.67 \pm 3.28^{\mathrm{a}}$
$255.33 \pm 1.45^{\mathrm{a}}$
$255.35 \pm 0.67^{\mathrm{a}}$
$244.67 \pm 2.60^{\mathrm{b}}$
$237.33 \pm 1.45^{\mathrm{c}}$
$204.01 \pm 2.50^{\mathrm{h}}$
$196.33 \pm 2.33^{\mathrm{i}}$
$196.33 \pm 2.98^{\mathrm{i}}$
$178.75 \pm 2.31^{\mathrm{j}}$
$170.67 \pm 2.46^{\mathrm{k}}$
$229.67 \pm 3.75^{\mathrm{d}}$
$220.33 \pm 1.45^{\mathrm{ef}}$
$220.35 \pm 0.65^{\mathrm{e}}$
$213.12 \pm 0.96^{\mathrm{fg}}$
$207.29 \pm 4.43^{\mathrm{gh}}$
$\mathbf{7 . 3 4 7 5}$

\begin{tabular}{c}
$\begin{array}{c}\text { Grain yield } \\
\left(\mathbf{t} \mathbf{~ h a}^{-1}\right)\end{array}$ \\
$7.51 \pm 0.11^{\mathrm{a}}$ \\
$6.78 \pm 0.02^{\mathrm{bc}}$ \\
$6.84 \pm 0.05^{\mathrm{bc}}$ \\
$6.47 \pm 0.11^{\mathrm{d}}$ \\
$6.16 \pm 0.07^{\mathrm{e}}$ \\
$5.37 \pm 0.00^{\mathrm{f}}$ \\
$4.91 \pm 0.18^{\mathrm{g}}$ \\
$4.93 \pm 0.18^{\mathrm{g}}$ \\
$4.27 \pm 0.10^{\mathrm{h}}$ \\
$3.74 \pm 0.11^{\mathrm{i}}$ \\
$6.97 \pm 0.05^{\mathrm{b}}$ \\
$6.56 \pm 0.05^{\mathrm{cd}}$ \\
$6.58 \pm 0.03^{\mathrm{cd}}$ \\
\hline $5.40 \pm 0.08^{\mathrm{f}}$ \\
$4.99 \pm 0.20^{\mathrm{g}}$ \\
$\mathbf{0 . 3 0 7 3}$
\end{tabular}

\section{Biological yield $\left(\mathrm{t} \mathrm{ha}^{-1}\right)$ \\ $21.82 \pm 0.02^{\mathrm{a}}$ \\ $21.07 \pm 0.17^{\mathrm{ab}}$ \\ $21.16 \pm 0.44^{\mathrm{ab}}$ \\ $20.16 \pm 0.25^{\text {cde }}$ \\ $19.34 \pm 0.10^{\mathrm{e}}$ \\ $18.29 \pm 0.13^{\mathrm{fg}}$ \\ $17.56 \pm 0.26^{\mathrm{g}}$ \\ $17.59 \pm 0.44^{\mathrm{g}}$ \\ $15.35 \pm 0.09^{\mathrm{h}}$ \\ $13.83 \pm 0.34^{\mathrm{i}}$ \\ $20.92 \pm 0.08^{\mathrm{bc}}$ \\ $20.20 \pm 0.15^{\mathrm{cd}}$ \\ $20.24 \pm 0.61^{\text {cd }}$ \\ $19.55 \pm 0.31^{\mathrm{de}}$ \\ $18.46 \pm 0.27^{\mathrm{f}}$ \\ 0.8288}

Means which followed by the same letters within a column are not significantly different at $P \leq 0.05 ; M_{1}=$ Ridge Sowing, $M_{2}=$ Flat Sowing, $M_{3}=$ Bed Sowing; $T_{0}=$ Control (two manual hoeings); $T_{1}=$ Mesotrion + atrazine @ $49.4 \mathrm{~g}$ a.i. ha $a^{-1}+494 \mathrm{~g}$ a.i. ha $\mathrm{a}^{-1}$ (Recommended dose $) ; T_{2}=$ Mesotrion + atrazine@ $39.52 \mathrm{~g}$ a.i. $\mathrm{ha}^{-1}+395.2 \mathrm{~g}$ a.i.ha $\mathrm{a}^{-1}+3 \%$ Urea $; T_{3}=$ Mesotrion + atrazine @ 29.64 g a.i. $h a^{-1}+296.4$ g a.i. ha $a^{-1}+3 \%$ Urea; $T_{4}=$ Mesotrion + atrazine @ 19.76 ga.i. ha $a^{-1}+197.6$ g a.i. ha $a^{-1}+3 \%$ Urea.

Those plots in which maize was planted with ridge sown technique and to manage weed interference two manual hoeings (15 and 30 DAS) were found most effective in control weed and exhibited longest cobs and maximal number of grains per cob, which was followed by ridge sown plots sprayed with Mesotrion + atrazine @ $39.52 \mathrm{~g}$ a.i. ha $^{-1}+395.2$ g a.i. $\mathrm{ha}^{-1}+3 \%$ Urea. Smallest cobs and least number of grains per cob were documented in those plots where maize was planted with flat sown scheme and sprayed with Mesotrion + Atrazine@19.76 g a.i. ha ${ }^{-1}+197.6$ g a.i. ha $^{-1}+3 \%$ Urea (Table 4).

Likewise, various sowing methods and weed control strategies significantly affected the thousand grain weight, economic yield and biological yield. Interaction between different sowing techniques and weed control practices was also found significant (Table 4). Highest values of thousand grain weight and economic and biological yield were recorded in those plots where maize was planted with ridge sown technique and to control weed infestation two manual hoeings were practiced after fifteen and thirty days of sowing followed by ridge sowing plots sprayed with Mesotrion + atrazine @ $39.52 \mathrm{~g}$ a.i. $\mathrm{ha}^{-1}+395.2$ g a.i. ha ${ }^{-1}+3 \%$ Urea. Moreover, combo of flat sowing with Mesotrion + atrazine @ $49.4 \mathrm{~g}$ a.i. ha ${ }^{-1}+494 \mathrm{~g}$ a.i. $\mathrm{ha}^{-1}$ and Mesotrion + atrazine @ $39.52 \mathrm{~g}$ a.i. $\mathrm{ha}^{-1}+395.2 \mathrm{~g}$ a.i. $\mathrm{ha}^{-1}+3 \%$ Urea were found statistically equal. Least values of 1000 gain weight, grain yield and biological yield were taken from flat sown plots sprayed with (Mesotrion + Atrazine @ 19.76 g a.i. $\mathrm{ha}^{-1}+197.6 \mathrm{~g}$ a.i. $\mathrm{ha}^{-1}+3 \%$ Urea). Discussing particularly about economic analysis, combo of ridge sowing method and two hand hoeings at 15 and 30 DAS are most effective as it gave maximum net return and high benefit to cost ratio (Table 4).

\section{Discussion}

Present study was designed to evaluate the weed inhibiting ability of different combinations of cultural and chemical weed control practices. It was the first time when efficacy of various sowing methods (ridge, bed and flat sowing) in combination with chemical (at reduced rate) and non-chemical weed control practices were evaluated simultaneously and their profitability was also assessed. Lowest weed population at 20 and 40 DAS was recorded in maize field planted under ridge sowing method and to control weeds two hand hoeings were practiced. Significant reduction in weed infestation in manual hoeing may be due to mechanical injury and partial eradication of weeds which suppressed them too strongly to compete with crop and chances of establishment of new weeds were reduced due to smothering effect of crop over weeds (Khan \& Haq, 2004). But manual hoeing was not practically feasible on large area due to acute weather during summer and shortage of labor, therefore other approaches should be adopted to deal with weeds for large areas.

Results of non-chemical technique (two hand hoeings) were best but statistically similar to ridge sown plots sprayed with Mesotrion + Atrazine@ $39.52 \mathrm{~g}$ a.i. $\mathrm{ha}^{-1}+395.2 \mathrm{~g}$ a.i. ha ${ }^{-1}+$ $3 \%$ Urea (Table 1). In present study, it was evident that dose of Felliston Gold can be reduced by using 3\% Urea as an 
adjuvant. Better weed control with reduced rate of herbicide may be due to better retention of herbicide on the leaves (Zadorozhny, 2004) and enhanced phytotoxicity (Borona et al., 2003).

Density of $T$. portulacastrum and $C$. rotundus was decreased when crop was planted on ridges than flat sowing. As crop sown on ridges, weed seeds may have been uncovered above soil surface while making seeds exposed to harsh weather ultimately suppressed the number of weed seeds germinated than flat sowing (Nadeem et al., 2013).

Significant reduction in weed numbers was the basic reason behind the least fresh and dry weight of weeds in those plots where maize was planted with ridge sown technique than the plantation of maize with bed and flat sowing. The interactive effect of chemical weed control treatments and different sowing methods on dry weight of weeds was non-significant; these findings are in line with the results of Maqbool et al. (2001), who found non-significant effect of interaction among chemical weed control treatments and different sowing methods on dry weight of weeds, the less weed dry weight in case of manual hoeing was due to less number of weeds and their fresh weight.

Significantly higher uptake of primary nutrients (NPK) by weeds in less weed control plots (bed and flat sown) clearly indicated the more competition of weed flora with maize plants. In ridge sown plots due to less weed density there was less weed-maize competition so more nutrients were accessible to crop and therefore stimulated greater absorption of nutrients and additional yield. These results verified by the findings of Jat et al. (2004) and Kanojia \& Nepalia (2006).

Effective weed control by non-chemical method (two hand hoeing's 30 and 60 DAS) was followed by the chemical control at reduced rate of herbicide (Mesotrion + atrazine @ $39.52 \mathrm{~g}$ a.i. $\mathrm{ha}^{-1}+395.2 \mathrm{~g}$ a.i. $\mathrm{ha}^{-1}+3 \%$ Urea) and less weedmaize competition for macro nutrients in plots planted with ridge sowing technique resulted in significantly greater yield and related. Likewise, Shinde et al. (2001) recorded significantly improved yield and related components in plots where weeds were below economic threshold level.

Less infestation of weeds resulted in higher uptake of nutrients, which indicates better development of root system in ridge sown plots resulted in better uptake of water and nutrients specially P (Chassot \& Richner, 2002) and ultimately greater grain weight, biological and grain yield. Reduction in infestation of weeds by proper use of herbicides also becomes the cause of plant height and biological yield of maize (Nalewaja et al., 2007).

Decreased doses of chemical herbicides by addition of adjuvants (Razzaq et al.,2012) also enhance biological yield, grain yield, harvest index and net profit for maize (Laosinwattana et al., 2012).

\section{Conclusion}

Plantation methods and herbicides at reduced rates with adjuvant application significantly affected the weed infestation and total grain production of autumn maize by influencing weed-maize competition. Autumn maize plantation with ridge sowing method and two hand hoeings at 15 and 30 DAS were found most effective to suppress the infestation of weeds and increased the yield of maize under agro-ecological conditions of Faisalabad, but manual hoeing has many limitations such as time consuming and laborious, only practical to a small area. On a large area due to shortage of labor and acute weather in June and July manual hoeing become almost mission impossible. Chemical control method is alternative tool and it is most efficient and effective by optimizing efficiency of herbicides at reduced rate by addition of adjuvant. Urea as an adjuvant increased the phytotoxic effect of herbicides applied at $20 \%$ less dose, and resulted outcomes were same as full herbicide dose used without adjuvant. Thus, plantation of autumn maize with ridge sown technique and to control weeds, spray at 20\% reduced dose of Felliston Gold 550Sc with 3\% urea application seems more feasible and practical.

\section{Conflict of interest}

Authors would hereby like to declare that there is no conflict of interests that could possibly arise.

\section{References}

Afzal I, Noor MA, Ahmad AH, Bakhtavar MA, Haq ZU (2015) Improvement of spring maize performance through physical and physiological seed enhancements. Seed Science \& Technology 43, 238-249.

Ahmad W, Noor MA, Afzal I, Bakhtavar MA, Nawaz MM, Sun X, Zhou B, Ma W, Zhao M (2016) Improvement of Sorghum Crop through Exogenous Application of Natural Growth-Promoting Substances under a Changing Climate. Sustainability 8: 1330. http://dx.doi.org/10.3390/su8121330

Alias A, Usman M, Ehsanllah, Warraich EA (2003) Effect of different phosphorus levels on growth and yield of two cultivars of maize. International Journal of Agriculture and Biology 5:142-147.

Amin M, Razzaq A, Rehmatullah, Ramzan M (2006) Effect of planting methods, seed density and nitrogen phosphorus (NP) fertilizer levels on sweet corn (Zea mays L.). Journal of Research Science 17:83-89.

Anonymous (2006) Crop Protection Handbook. Meister Publishing Company, Ohio.

Bakht J, Shafi M, Rehman H, Uddin R, Anwar S (2011) Effect of planting methods on growth, phenology and yield of maize varieties. Pakistan Journal of Botany 43: 1629-1633. 
Bakhtavar, MA, Afzal I, Basra SMA, Ahmad AH, Noor MA (2015) Physiological Strategies to Improve the Performance of Spring Maize (Zea mays L.) Planted under Early and Optimum Sowing Conditions. PLoS ONE 10: e0124441. doi:10.1371/journal.pone.0124441

Borona V, Zadorozhny V, Postolovskay T (2003) The influence of adjuvant and efficacy of graminicides in soybean and nicosulfuron in maize. Proceeding of the $2^{\text {nd }}$ weed conference, Sarajevo, Bosina and Herzogovin 4: 151-155.

Bremner JM (1965) Inorganic forms of nitrogen. Methods of soil analysis. Part 2. Chemical and microbiological properties, Iowa State University Ames press, USA, 1179-1237.

Bunting JA, Sprague CL, Riechers DE (2004) Proper adjuvant selection for foramsulfuron activity. Crop Protection 23: 361 366.

Chassot A, Richner W (2002) Root characteristics and phosphorus uptake of maize seedlings in a bilayered soil. Agronomy Journal 94: 118-127. DOI:10.2134/agronj2002.1180.

Chikoye D, Schulz S, Ekeleme F (2004) Evaluation of integrated weed management practices for maize in the northern Guinea savanna of Nigeria. Crop Protection 23: 895900.http://dx.doi.org/10.1016/j.cropro.2004.01.013.

Devendra R, Umamahesh V, Prashad, TVR, Prashad TG, Asha ST, Ashok(2004) Influence of surfactants on efficacy of different herbicides in control of Cyperus rotundus and Oxalis latifolia. Current Sciences 86: 8-86.

FAO (Food and Agricultural Organization) (2011) FAOSTAT database for agriculture. Available online athttp://faostat.fao.org/faostat/collection?subset=agriculture access on 25 july, 2015.

Getmanetz AY, Kramarev SM, Vittsenko VP, Bovykin BA, Tishkina NS, Matrosov AS (1991) Chemical compatibility of Zhku 10-34-0, KAS-28 and herbicides and their combine use in intensive maize growing technology. Agrokhimiya 11:3844.

Green JM, Beestman GB (2007) Recently patented and commercialized formulation and adjuvant technology. Crop Protection 26: 320-327. DOI: http://dx.doi.org/10.1016/j.cropro.2005.04.018.

Jat ML, Pal SS, Subba Rao AVM, Sirohi K, Sharma SK, Grupta RK (2004) Laser land levelling: the precursor technology for resource conservation in irrigated ecosystem of India. In; National Conference on Conservation Agriculture: Conserving Resources and Enhancing Productivity, Conservation Agriculture-Status and Prospects, 22-23 September, 2004, CASA, New Delhi, 145-154.
Kanojia Y, Nepalia V (2006) Effect of chemical weed control on nutrient uptake by wheat and associated weeds. Agricultural Science Digest 26: 141-143

Khan M, Haq N (2004) Weed control in maize (Zea mays L) with pre and post emergence herbicides. Pakistan Journal of Weed Science Research 10: 39-46.

Laosinwattana C, Teerarak M, Charoenying P (2012) Effects of Aglaia odorata granules on the seedling growth of major maize weeds and the influence of soil type on the granule residue's efficacy. Weed Biology and Management 12: 117122.DOI: 10.1111/j.1445-6664.2012.00444.x.

Maqbool MM, Tanveer A, Ali A, Ahmad R (2001) Effect of sowing methods and herbicides on weeds and yield of cotton. Pakistan Journal of Botany 33: 383-387.

Memon RA, Bhatti G R, Khalid S, Soomro R, Ahmed S (2007) A survey of weeds found in cotton fields of the Khairpur district, Sindh, Pakistan. Pakistan Journal of Botany 39: 22652274

Nadeem MA, Idrees M, Ayub M, Tanveer A, Mubeen K (2013) Effect of different weed control practices and sowing methods on weeds and yield of cotton. Pakistan Journal of Botany 45: 1321-1328.

Nalewaja DJ, Matysiak R, Hanmker P (2007) Change in efficacy of glyphosate by adjuvant. Weed Technology 21: 662681.

Oerke EC, Dehne HW (2004) Safeguarding Production losses in major crops and the role of crop production. Crop Protection 23: 275- 285.2 DOI: http://dx.doi.org/10.1016/j.cropro.2003.10.001

Pacanoski Z (2007) Herbicide use: Benefit for society as a whole. Pakistan Journal of Weed Science Research13:135-147.

Razzaq A, Cheema ZA, Jabran K, Hussain M, Farooq M, Zafar M (2012) Reduced herbicide doses used together with allelopathic sorghum and sunflower water extracts for weed control in wheat. Journal of Plant Protection Research 52: 281-285

Shinde SH, Kolage AK, Bhilare RL (2001) Effect of weed control on growth and yield of maize. Journal of Maharashtra Agricultural Universities 26: 212-213.

Ssango F, Balitenda M (2003) Effect of application of which weed control options on performance of two maize varieties in Uganda. Muarik Bulletin 6: 83-88.

Steel RGD, Torrie JH, Dicky DA (1997) Principles and Procedures of Statistics-a Biometrical Approach. 3rd Ed. McGraw Hill Book International Co., Singapore, pp: 204-227. 
Ullah A, Khaliq A, Riaz A, Noor MA, Waqas MA, Zain M, Fiaz S, Ashraf U, Nawaz A (2017) Seed pre-treatment and planting geometry positively influence herbicide efficacy in wheat (Triticum aestivum L.). Planta Daninha (In Press)

Wolf B (1982) A comprehensive systems of leaf analysis and its use for diagnosing crop nutrients status. Communications in
Soil Science and Plant Analysis 13: 1035-1059. DOI: http://dx.doi.org/10.1080/00103628209367332.

Zadorozhny V (2004) Herbicide based strategies for maize to prevent development of resistance in weeds in Ukraine. Weed Management Proceedings of 14th Australian weed conference, WaggzaWagga, New south Wales Australia 6-9 September, 2004 pp. 290-293. 